\title{
The Phase 3 COU-AA-302 Study of Abiraterone Acetate Plus Prednisone in Men with Chemotherapy-naiive Metastatic Castration-resistant Prostate Cancer: Stratified Analysis Based on Pain, Prostate-specific Antigen, and Gleason Score
}

\author{
Kurt Miller $^{a, *}$, Joan Carles ${ }^{b}$, Jürgen E. Gschwend ${ }^{c}$, Hendrik Van Poppel ${ }^{d}$, Joris Diels ${ }^{e}$, \\ Sabine D. Brookman-May ${ }^{f, g, * *}$
}

${ }^{a}$ Department of Urology, Charité Berlin, Berlin, Germany; ${ }^{\mathrm{b}}$ Valld'Hebron Institute of Oncology, Valld'Hebron University Hospital, Barcelona, Spain; ${ }^{\mathrm{c}}$ Department of Urology, Technical University of Munich, Munich, Germany; ${ }^{\mathrm{d}}$ Katholieke Universiteit Leuven, Leuven, Belgium; ${ }^{\mathrm{e} J a n s s e n ~ E M E A ~ H E M A R, ~ B e e r s e, ~ B e l g i u m ; ~}$ ${ }^{\mathrm{f}}$ Department of Urology, Ludwig-Maximilians University (LMU) Munich, Munich, Germany; ${ }^{\mathrm{g}}$ Janssen Research E Development, Los Angeles, California, USA

\section{Article info}

Article history:

Accepted August 29, 2017

Associate Editor:

Giacomo Novara

Statistical Editor:

Andrew Vickers

Keywords:

Abiraterone acetate

Metastatic castration-resistant

prostate cancer

Stratification

Prognostic discrimination

Predictive parameters

\begin{abstract}
Background: In the COU-AA-302 study (NCT00887198), abiraterone acetate plus prednisone (AAP) significantly improved outcomes in patients with metastatic castrationresistant prostate cancer ( $\mathrm{MCRPC}$ ) versus prednisone alone. Baseline clinical parameters predicting that treatment response could help inform clinical decisions were explored. objective: To identify patients who derive the greatest clinical benefit from AAP treatment.

Design, setting, and participants: A total of 1088 mCRPC patients treated with either AAP or prednisone in the first-line setting in COU-AA-302 were included in this post hoc analysis.

Intervention: Abiraterone acetate $1000 \mathrm{mg}$ daily versus placebo, both plus prednisone $10 \mathrm{mg}$ daily.

Outcome measurements and statistical analysis: Univariate and multivariable Cox regression analyses were performed, including clinical and pathological parameters for the primary end points overall survival (OS) and radiographic progression-free survival (rPFS), and secondary study end points. Tumor-associated baseline parameters independently impacting OS were applied to stratify patients according to possible treatment effects.

Results and limitations: Baseline prostate-specific antigen (PSA), tumor-related pain as assessed by the Brief Pain Inventory-Short Form (BPI-SF), and Gleason score (GS) at primary diagnosis were identified as tumor-associated variables that independently impacted OS. AAP significantly improved outcomes versus prednisone in both group 1 (BPI-SF $0-1$ and $\mathrm{PSA}<80 \mathrm{ng} / \mathrm{ml}$ and GS $<8 ; p=0.006$; hazard ratio [HR]: 0.61) and group 2 (BPI-SF 2-3 and/ or PSA $\geq 80 \mathrm{ng} / \mathrm{ml}$ and/or GS $\geq 8 ; p=0.03 ; \mathrm{HR}: 0.84$ ). The differences observed for treatment effects between groups 1 and 2 for OS (HR: 0.61 vs 0.84 ), rPFS (HR: 0.41 vs 0.59 ), and time to chemotherapy (HR: 0.64 vs 0.71 ) were not statistically significant.
\end{abstract}


Conclusions: AAP significantly improved outcomes in mCRPC patients compared with prednisone alone regardless of baseline pain and PSA level, and GS at primary diagnosis with no significant differences between observed treatment effects in groups 1 and 2 .

Patient summary: Treatment with abiraterone acetate and prednisone (compared with treatment with prednisone only) for metastatic castration-resistant prostate cancer increased survival in all patients in the study regardless of pain, prostate-specific antigen levels at the start of treatment, and Gleason score at primary diagnosis.

(c) 2017 European Association of Urology. Published by Elsevier B.V. All rights reserved.

\section{Introduction}

The therapeutic landscape in metastatic castration-resistant prostate cancer ( $\mathrm{MCRPC}$ ) has expanded in recent years with the introduction of several novel anticancer drugs prolonging overall survival (OS), including the CYP17 inhibitor abiraterone acetate (AA), the androgen-receptor antagonist enzalutamide, the radioisotope alpharadin (radium-223), the taxane cabazitaxel, and the vaccine sipuleucel$\mathrm{T}$ (marketing authorization withdrawn in the European Union) [1-3]. These treatment options exploit different mechanisms of action; therefore, different biomarkers and prognostic variables are most relevant to measuring and predicting treatment response for different agents.

Independent predictors of OS previously identified include, but are not limited to, tumor-associated markers such as prostate-specific antigen (PSA) and Gleason score (GS), bone turnover markers such as alkaline phosphatase (ALP), patient-related factors such as cancer-related pain and performance status, metastatic distribution (eg, presence or absence of visceral metastases), and laboratory parameters, including hemoglobin, lactate dehydrogenase (LDH), albumin, and neutrophil-to-lymphocyte ratio $[1,4]$. Further research into predictors of patient response to different treatments would benefit physicians, supporting evidence-based clinical decision making on initial treatment selection and optimal sequential treatments [1].

AA in combination with prednisone (AAP) was initially approved in 2011 for the treatment of MCRPC patients after chemotherapy, and subsequently for the first-line treatment of asymptomatic or mildly symptomatic chemotherapy-naïve patients based on results from the pivotal phase 3 COU-AA-302 study (NCT00887198) [5,6]. In COU-AA-302, AAP significantly improved median radiographic progression-free survival (rPFS) and median OS versus placebo plus prednisone (PP) [6], and delayed clinical deterioration and initiation of chemotherapy [5]. Similar survival benefit from AAP was observed across several predefined patient subgroups, regardless of baseline Eastern Cooperative Oncology Group performance status(ECOG PS) (0 vs 1), tumor-associated pain assessed by the Brief Pain InventoryShort Form (BPI-SF) score (0-1 vs 2-3), metastatic sites (bone only or bone and other sites), age $(<65, \geq 65$, or $\geq 75$ $\mathrm{yr}$ ), and PSA, LDH, and ALP levels [5,6].

More in-depth analysis of outcomes across patient subgroups may reveal differential treatment effects and point to clinical characteristics with prognostic value in predicting treatment response. Here, we report a post hoc analysis of COU-AA-302 with patients stratified according to baseline characteristics, with the aims of identifying patients deriving the greatest clinical benefit from AAP treatment and describing prostate cancer-related parameters identified at initiation of therapy to inform further clinical decisions.

\section{Patients and methods}

COU-AA-302 was a phase 3, double-blind, placebo-controlled, randomized clinical trial in which 1088 asymptomatic or mildly symptomatic chemotherapy-naïve $\mathrm{mCRPC}$ patients received prednisone $5 \mathrm{mg}$ twice daily plus AA $1000 \mathrm{mg}$ once daily or placebo (Fig. 1). Details of the study design have been reported previously [5]. Patients had MCRPC after PSA progression according to Prostate Cancer Clinical Trials Working Group 2 criteria [7] or radiographic progression under ongoing androgendeprivation therapy, serum testosterone levels $<50 \mathrm{ng} / \mathrm{dl}$, ECOG PS grade 0 or 1 , no or mild tumor-related pain (BPI-SF scores of $0-1$ [asymptomatic] or 2-3 [mildly symptomatic]), and hematological and chemical laboratory values that met predefined criteria. Patients with visceral metastases or those who had received previous ketoconazole therapy lasting $>7 \mathrm{~d}$ were excluded.

This stratification analysis utilized the final dataset from the COUAA-302 study. Median follow-up duration for patients alive at the end of follow-up was $49.2 \mathrm{mo}$. As a first step, baseline demographic, clinical, and laboratory parameters were analyzed for association with OS in the overall study population using univariate and multivariable Cox

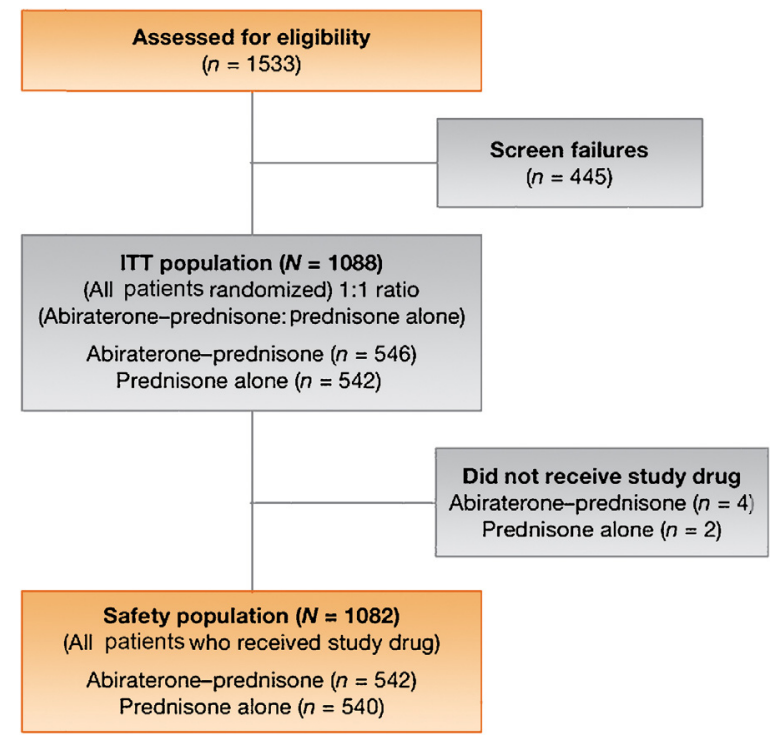

Fig. 1 - CONSORT study flow diagram. CONSORT = Consolidated Standards of Reporting Trials; ITT = intention to treat. 
Table 1 - Univariate and multivariable Cox regression analysis for the end point overall survival

\begin{tabular}{|c|c|c|c|c|}
\hline & \multicolumn{2}{|c|}{ Univariate analysis } & \multicolumn{2}{|c|}{ Multivariable analysis } \\
\hline & HR (95\% CI) & $p$ value & $\mathrm{HR}(95 \% \mathrm{CI})$ & $p$ value \\
\hline Tumor-related pain (BPI-SF score $2-3$ vs $0-1$ ) & $1.65(1.40-1.94)$ & $<0.001$ & $1.30(1.10-1.53)$ & 0.002 \\
\hline PSA, ng/ml (log) & $1.27(1.20-1.33)$ & $<0.001$ & $1.16(1.10-1.22)$ & $<0.001$ \\
\hline ECOG PS 1 vs 0 & $1.36(1.16-1.60)$ & 0.002 & $1.17(0.98-1.39)$ & 0.08 \\
\hline Gleason score $<8$ vs $\geq 8$ & $0.81(0.71-0.94)$ & 0.005 & $0.78(0.68-0.91)$ & 0.001 \\
\hline $\mathrm{Hb}, \mathrm{g} / \mathrm{dl}$ & $0.82(0.77-0.87)$ & $<0.001$ & $0.92(0.86-0.98)$ & 0.006 \\
\hline LDH, IU/l (log) & $4.54(3.41-6.04)$ & $<0.001$ & $2.37(1.73-3.24)$ & $<0.001$ \\
\hline ALP, IU/l (log) & $1.82(1.63-2.02)$ & $<0.001$ & $1.34(1.19-1.52)$ & $<0.001$ \\
\hline Age, yr & $1.02(1.01-1.03)$ & $<0.001$ & $1.01(1.00-1.02)$ & 0.007 \\
\hline
\end{tabular}

proportional hazard regression models. Age and hemoglobin were modeled as continuous variables; PSA, LDH, and ALP were modeled as continuous variables on a logarithmic scale, and pain score, ECOG PS, and GS as categorical variables.

For parameters with an independent prognostic impact on OS in the multivariable Cox regression analysis, those specifically associated with prostate cancer were analyzed further to assess whether any differential treatment effects across subgroups could be affirmed. The COU-AA-302 study population was then stratified into two groups based on a combination of the identified factors. For continuous variables, the cutoff points for assigning patients to group 1 or 2 were determined based on values providing the greatest differentiation in hazard ratio (HR) for OS between groups, to support the objective of identifying a subgroup of patients deriving the greatest clinical benefit from AAP treatment. The treatment effect of AAP versus PP was analyzed within each stratified group. The end points OS, rPFS, time to chemotherapy use, time to opiate use, and time on treatment were estimated using the Kaplan-Meier method and analyzed by Cox proportional hazard regression.

\section{Results}

\subsection{Univariate and multivariable analyses of oS}

Univariate analysis identified several variables with a significant association with OS (Table 1). Significance was maintained on multivariable analysis for baseline BPI-SF score, PSA, hemoglobin, LDH, ALP, age, and GS at primary diagnosis (Table 1 ).

\subsubsection{Stratified analysis}

Of those parameters with a significant impact on OS on multivariable analysis, parameters directly associated with prostate cancer (ie, tumor-related pain/BPI-SF, PSA, and GS) were selected for additional evaluation of potential predictive relevance for the treatment efficacy of AAP. Other parameters, including LDH, ALP, and hemoglobin, had an independent impact on OS; however, as these are not prostate cancer-specific markers with the potential to change for reasons other than tumor progression, they were not considered for additional evaluation and stratification.

Pain, PSA, and GS were used to stratify patients into two groups: group 1 patients were asymptomatic, reporting no tumor-related pain (BPI-SF 0-1), PSA $<80 \mathrm{ng} / \mathrm{ml}$, and GS $<8$; group 2 patients had mild pain (BPI-SF $\geq 2$ ) and/or PSA $\geq 80$ $\mathrm{ng} / \mathrm{ml}$ and/or $\mathrm{GS} \geq 8$. The BPI-SF cut-off allowed patients to be categorized into those with no and mild pain; the GS cutoff categorizes patients with poorly differentiated versus

Table 2 - Baseline characteristics of patients in the overall study population and in groups 1 and 2

\begin{tabular}{|c|c|c|c|}
\hline Characteristic & Overall cohort ( $n=1088)$ & Group $1(n=264)$ & Group $2(n=824)$ \\
\hline BPI-SF score $0-1, \%$ & 66 & 100 & 55 \\
\hline $\begin{array}{l}\text { Median PSA, ng/ml } \\
(\mathrm{IQR})\end{array}$ & $\begin{array}{l}39.5 \\
\text { (IQR: 15.6-107) }\end{array}$ & $\begin{array}{l}24.1 \\
\text { (IQR: 11.1-41.4) }\end{array}$ & $\begin{array}{l}58.5 \\
\text { (IQR: 18.6-145) }\end{array}$ \\
\hline Gleason score $\geq 8, \%$ & 48 & 0 & 63 \\
\hline ECOG PS $1, \%$ & 24 & 17 & 37 \\
\hline $\begin{array}{l}\text { Median Hb, g/dl } \\
\text { (IQR) }\end{array}$ & $\begin{array}{l}13.1 \\
\text { (IQR: 12.2-13.8) }\end{array}$ & $\begin{array}{l}13.3 \\
\text { (IQR: 12.6-14) }\end{array}$ & $\begin{array}{l}13.0 \\
\text { (IQR: 12.1-13.7) }\end{array}$ \\
\hline $\begin{array}{l}\text { Median LDH, IU/l } \\
(\mathrm{IQR})\end{array}$ & $\begin{array}{l}185 \\
\text { (IQR: 163-214) }\end{array}$ & $\begin{array}{l}180 \\
\text { (IQR: 162-202) }\end{array}$ & $\begin{array}{l}187 \\
\text { (IQR: 164-218) }\end{array}$ \\
\hline $\begin{array}{l}\text { Median ALP, IU/l } \\
\text { (IQR) }\end{array}$ & $\begin{array}{l}91 \\
\text { (IQR: 69-136) }\end{array}$ & $\begin{array}{l}79 \\
\text { (IQR: 64-102) }\end{array}$ & $\begin{array}{l}97 \\
\text { (IQR: 72-151) }\end{array}$ \\
\hline Median age, yr & $\begin{array}{l}70 \\
\text { (IQR: 64-77) }\end{array}$ & $\begin{array}{l}70 \\
\text { (IQR: 64-76.5) }\end{array}$ & $\begin{array}{l}71 \\
\text { (IQR: 64-77) }\end{array}$ \\
\hline
\end{tabular}


A

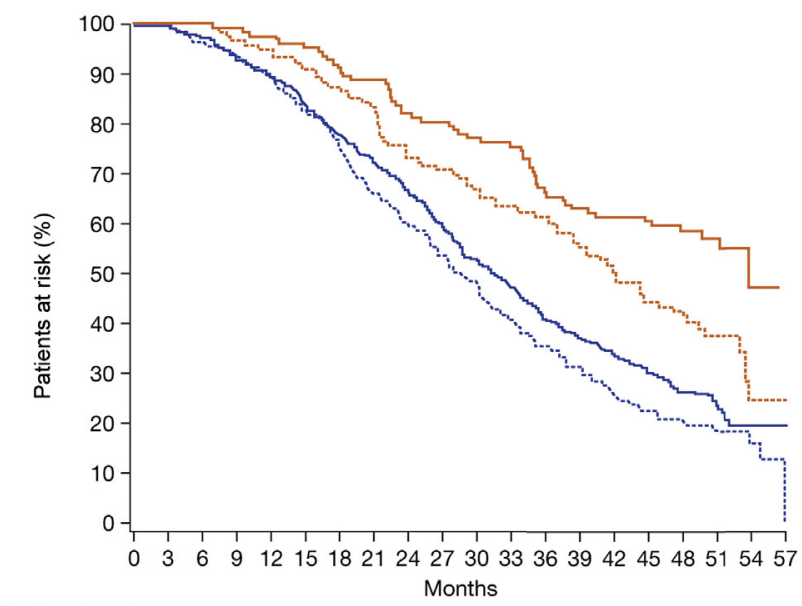

Number of events

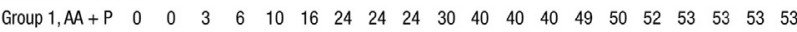
$\begin{array}{llllllllllllllllllllll}\text { Group 1, } \mathrm{P} & 0 & 0 & 0 & 0 & 11 & 15 & 23 & 37 & 40 & 45 & 49 & 51 & 51 & 62 & 62 & 74 & 78 & 79 & 82 & 83\end{array}$

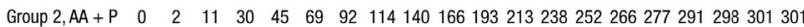

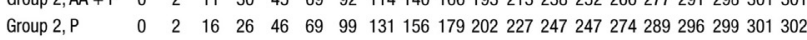

\section{C}

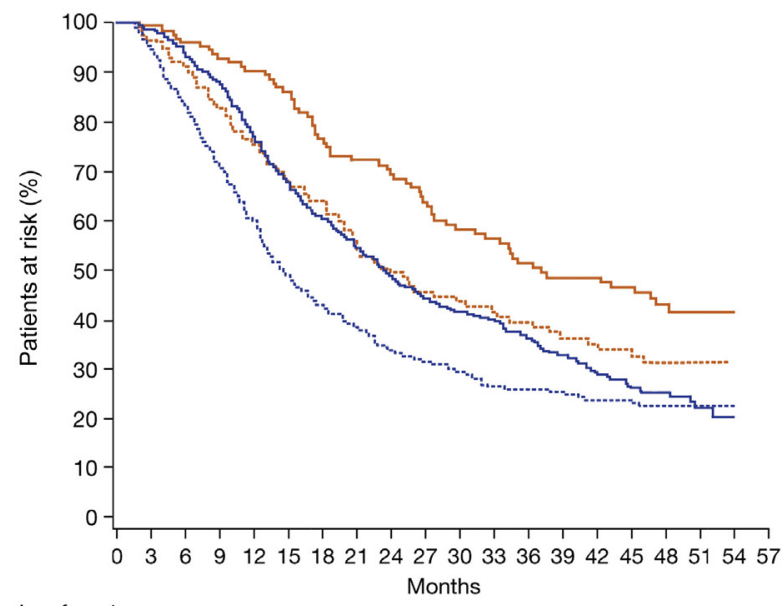

Number of events

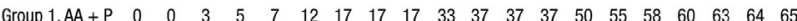
$\begin{array}{lllllllllllllllllllll}\text { Group 1, } \mathrm{P} & 0 & 0 & 11 & 11 & 29 & 34 & 44 & 52 & 63 & 65 & 70 & 70 & 70 & 74 & 74 & 77 & 80 & 82 & 82 & 82\end{array}$

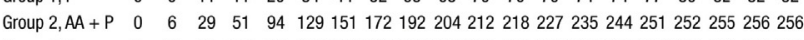
Group 2, P $\quad 0 \quad 22 \quad 66111151189210223239244249256258258262264264264264264$
B

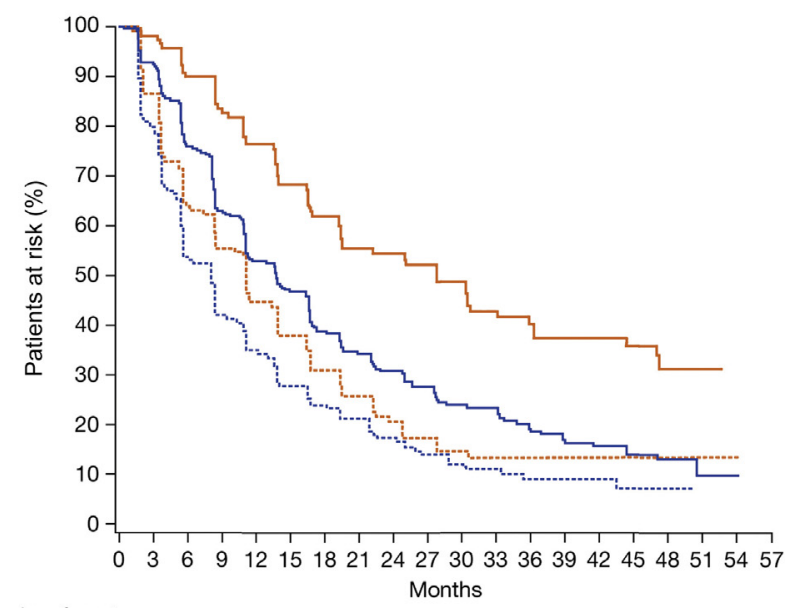

Number of events

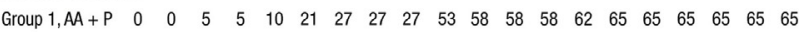
$\begin{array}{lllllllllllllllllllll}\text { Group 1, } \mathrm{P} & 0 & 0 & 36 & 36 & 58 & 70 & 89 & 93 & 98 & 99 & 99 & 99 & 99 & 99 & 99 & 99 & 99 & 99 & 99 & 99\end{array}$ Group 2, AA + P $0030 \quad 94142175192214225237247254258265266267267267267267267$ Group 2, P $\quad 0 \quad 76165198217232242254258262264264265265265265265265265265$

\section{D}

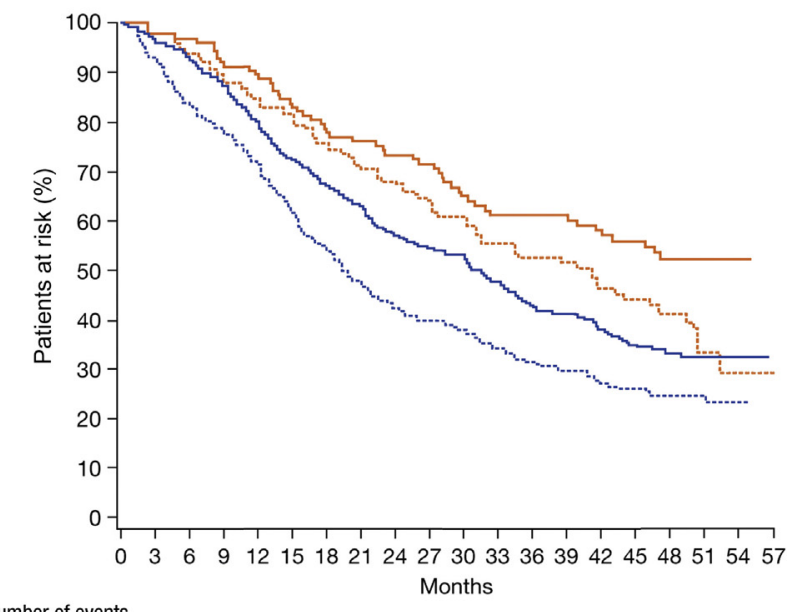

Number of events

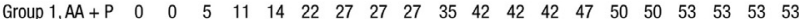
$\begin{array}{lllllllllllllllllllll}\text { Group 1, } P & 0 & 0 & 9 & 9 & 22 & 28 & 34 & 39 & 43 & 48 & 51 & 56 & 56 & 60 & 60 & 67 & 69 & 72 & 73 & 73\end{array}$

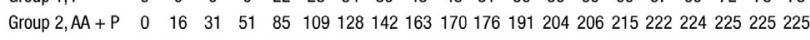
Group 2, P $\quad 0 \quad 31166 \quad 87111144172192207214220229236236244246248249249249$

Group 1, AA + P $(n=124)$

Group 1, P $(n=140)$

Group 2, AA + P (n= 422) Group 2, $\mathrm{P}(n=402)$

Fig. 2 - Kaplan-Meier analysis of time-to-event end points with abiraterone acetate plus prednisone and prednisone: (A) overall survival; (B) radiographic progression-free survival. $(C)$ time to chemotherapy; and $(D)$ time to opiate use. $A A=$ abiraterone acetate; $P=$ prednisone.

well or moderately differentiated tumors. The cutoff of $80 \mathrm{ng} / \mathrm{ml}$ for PSA was selected due to the largest differentiation of HR for OS between groups.

In total, 824 patients met the criteria for group 2, based on higher levels/scores in at least one of the three stratification parameters, while approximately one-quarter were asymptomatic and had lower PSA levels and GS (group $1 ; n=264)$. Patients in groups 1 and 2 were evenly distributed across treatment arms: in the AAP arm $(n=546), 23 \%(n=124)$ and $77 \%(n=422)$ of patients were in groups 1 and 2 , respectively; in the PP arm $(n=542)$, the proportions were 26\% $(n=140)$ and 74\% $(n=402)$, respectively. Baseline characteristics for both groups are shown in Table 2 .
In both groups, treatment with AAP significantly prolonged median OS, rPFS, time to chemotherapy use, time to opiate use, and treatment duration versus PP (Fig. 2 and Table 3). The median OS was 53.6 versus 41.8 mo (HR: $0.61 ; 95 \%$ confidence interval $[\mathrm{CI}], 0.43-0.87 ; p=0.006)$ in group 1 compared with 31.2 versus 28.4 mo (HR: $0.84 ; 95 \%$ CI, $0.72-0.99 ; p=0.03)$ in group 2 . Respective results for rPFS were 27.6 versus 11.1 mo (HR: 0.41 ; 95\% CI, 0.30-0.57; $p<0.001$ ) compared with 13.7 versus 8.2 mo (HR: $0.59 ; 95 \%$ CI, 0.50-0.70; $p<0.001)$. Median time to chemotherapy was 37.0 versus 24.3 mo (HR: $0.64 ; 95 \%$ CI, 0.46-0.89; $p=0.007$ ) in group 1 compared with 23.3 versus 14.5 mo (HR: $0.71 ; 95 \% \mathrm{CI}, 0.60-0.85 ; p<0.001$ ) in group 2 . The 
Table 3 - Analysis of time-to-event end points with abiraterone acetate plus prednisone and prednisone plus placebo within groups 1 and 2

\begin{tabular}{|c|c|c|c|c|}
\hline & \multicolumn{2}{|c|}{ Group 1} & \multicolumn{2}{|c|}{ Group 2} \\
\hline & $\mathrm{AAP}(n=124)$ & PP $(n=140)$ & $\mathrm{AAP}(n=422)$ & $\mathrm{PP}(n=402)$ \\
\hline \multicolumn{5}{|l|}{ Overall survival } \\
\hline Median, mo (IQR) & 53.6 (33.7-NR) & $41.8(23.4-53.6)$ & $31.2(19.4-50.4)$ & $28.4(17.9-41.9)$ \\
\hline Number of events $(n)$ & 53 & 83 & 301 & 302 \\
\hline $\mathrm{HR}(95 \% \mathrm{CI})$ & $0.61(0.43-0.87)$ & & $0.84(0.72-0.99)$ & \\
\hline$p$ value & 0.006 & & 0.03 & \\
\hline \multicolumn{5}{|l|}{ Radiographic PFS } \\
\hline Median, mo (IQR) & 27.6 (13.6-NR) & $11.1(3.7-21.8)$ & $13.7(7.4-27.7)$ & $8.2(3.5-16.6)$ \\
\hline Number of events $(n)$ & 65 & 99 & 267 & 265 \\
\hline $\mathrm{HR}(95 \% \mathrm{CI})$ & $0.41(0.30-0.57)$ & & $0.59(0.50-0.70)$ & \\
\hline$p$ value & $<0.001$ & & $<0.001$ & \\
\hline \multicolumn{5}{|l|}{ Time to chemotherapy use } \\
\hline Median, mo (IQR) & $37.0(18.3-54.7)$ & 24.3 (11.7-NR) & $23.3(12.5-48.2)$ & $14.5(8-39.2)$ \\
\hline Number of events $(n)$ & 65 & 82 & 256 & 264 \\
\hline $\mathrm{HR}(95 \% \mathrm{CI})$ & $0.64(0.46-0.89)$ & & $0.71(0.60-0.85)$ & \\
\hline$p$ value & 0.007 & & $<0.001$ & \\
\hline \multicolumn{5}{|l|}{ Time to opiate use } \\
\hline Median, mo (IQR) & NR (22.9-NR) & 41.0 (18.2-NR) & 30.5 (13.5-NR) & $19.3(10.6-46)$ \\
\hline Number of events $(n)$ & 53 & 73 & 225 & 249 \\
\hline $\mathrm{HR}(95 \% \mathrm{CI})$ & $0.69(0.48-0.99)$ & & $0.70(0.59-0.84)$ & \\
\hline$p$ value & 0.04 & & 0.0001 & \\
\hline \multicolumn{5}{|l|}{ Treatment duration } \\
\hline Median, mo & 20.4 & 11.2 & 12.3 & 7.2 \\
\hline $\mathrm{HR}(95 \% \mathrm{CI})$ & $0.41(0.31-0.54)$ & & $0.54(0.46-0.62)$ & \\
\hline$p$ value & $<0.001$ & & $<0.001$ & \\
\hline
\end{tabular}

interaction effects based on subgroup stratification by PSA, pain, and GS were not significantly different (group 1: $p=0.12$; group $2: p=0.74)$.

\section{Discussion}

This post hoc analysis of the COU-AA-302 study revealed that both patient groups achieved significantly improved outcomes with AAP versus PP, including improvements in OS and rPFS as well as in secondary study end points, regardless of pain and PSA levels at study entry and GS at primary diagnosis. While OS, rPFS, and time to chemotherapy and opiate use were longer in asymptomatic mCRPC patients, defined as patients with lacking or very minimal prostate cancer-related pain, PSA levels $<80 \mathrm{ng} / \mathrm{ml}$ at the start of treatment and a GS of $<8$ at primary diagnosis compared with patients with higher PSA levels, pain at the start of treatment, or a higher GS at primary diagnosis, no statistically significant different treatment interactions could be confirmed between both groups.

A prognostic index model developed in a previous analysis of data from COU-AA-302 identified the presence of more bone metastases ( $\geq 10$ vs $<10$ ), LDH or ALP levels above the upper limit of normal, and a higher BPI-SF score (2-3 vs $0-1)$ as risk factors for worse prognosis in this patient population [8]. The current study results were consistent with this prognostic index model in identifying LDH, ALP, and tumor-related pain as having a significant association with OS, as well as PSA, GS, hemoglobin, and age. Since parameters such as LDH, ALP, and hemoglobin are nonspecific and could change for reasons besides tumor progression, we focused on tumor-related parameters to stratify patients and identify predictors of treatment response. This post hoc analysis was also consistent with previous analyses of predefined subgroups [5,6] showing that that AAP was effective across the spectrum of patients participating in this trial.

While patients with lower levels of PSA and tumorrelated pain at the start of treatment and a lower GS at primary diagnosis had enhanced OS, rPFS, and secondary end points in comparison with those with higher PSA or pain levels or a higher GS, no significant difference was finally found in treatment effects between both patient groups. Hence, based on the results of this study, conclusions concerning treatment decision making and timing of treatment initiation in routine clinical practice cannot be drawn. Nonetheless, potential benefits by initiation of first-line treatment at a time point when pain and PSA elevation are limited to both delay the requirement for chemotherapy and improve survival outcomes are supported by the results of further studies and analyses. For example, in the PREVAIL study investigating enzalutamide in patients with chemotherapy-naïve mCRPC, patients in the placebo arm had rapid progression with a median 2.8 mo to PSA progression only [9].

The stratification parameters identified in the present analysis may have varying degrees of value in determining patient prognoses. Cancer-related pain has been reported to be prognostic in $\mathrm{MCRPC}$, independent of treatment, and may indicate the presence of more advanced disease $[10,11]$. There is also evidence that patient-reported parameters such as cancer pain might be more reliable than laboratory parameters for outcome prediction [12,13]. 
PSA elevation has been shown in some studies to be associated with worse outcomes when considered as an independent prognostic marker $[4,10,14]$. As a predictive indicator of treatment response, lower baseline PSA was associated with better survival outcomes in trials of sipuleucel-T [15] and AAP [16], but not enzalutamide [17]. In a post hoc analysis of the COU-AA-302 study in which patients were stratified into quartiles according to baseline PSA, radiographic progression and mortality risk decreased with decreasing baseline PSA, and the relative benefit of AAP versus PP was greatest in patients with lower baseline PSA [16]. Low baseline PSA also predicted longterm treatment response to AAP as second-line treatment after prior docetaxel treatment [18]. Similarly, patients in the lowest quartile for baseline PSA derived greatest benefit from sipuleucel-T treatment in the IMPACT study [15]. Accordingly, in a previous interim analysis of the COU-AA-302 trial, it has been shown that patients with low PSA values and no tumor-related pain had a significantly better clinical treatment effect than patients with advanced PSA and pain levels [19]. In contrast, in the AFFIRM study evaluating the efficacy of enzalutamide following docetaxel treatment, analysis by baseline PSA quartile found that the treatment effect on OS was consistent regardless of baseline PSA levels [17]. In mCRPC patients receiving enzalutamide as first-line treatment in the PREVAIL study, subgroup analyses showed no difference in survival outcomes for those with baseline PSA above versus below the median [9].

PSA kinetics (eg, rate of doubling from baseline) [20] and change in PSA during the first few months of treatment [21] may also be useful indicators of response to AAP; monitoring these parameters following initiation of treatment may help guide decisions on whether, or at which point, switching to the next treatment is indicated. PSA subforms (free PSA and pro-PSA, in addition to total PSA) may also be useful indicators of early response to AAP [22].

GS may have reduced prognostic significance in MCRPC compared with earlier disease, although a higher GS, which might increase during the natural history of the disease, has been associated with shorter OS [1,23]. There are, however, several considerations when analyzing the predictive or prognostic value of GS in clinical trials and routine clinical practice in mCRPC patients. GS is regularly based on biopsy taken at initial diagnosis or tissue from radical prostatectomy; this may have been several years prior to study entry, during which time histological changes, including development to a higher GS, may have occurred such that the GS does not reflect the true histology at the time of study treatment initiation. Furthermore, it is well known that there is a lack of concordance between biopsy GS and definitive GS in tissue obtained in radical prostatectomy [24]. In the COU-AA-302 study, half of the samples were from biopsy and half from prostatectomy specimens. Furthermore, the grading system for GS was amended in 2005; therefore, samples taken before 2005 (which applies for some patients in the COU-AA-302 study) may be scored differently from those taken more recently and graded according to the International Society of Urological Pathology modified Gleason grading system [25]. Considerable inter- and intraobserver variation in the assessment of GS has also been noted [26]. In a previously published analysis, GS alone ( $<8 \mathrm{vs} \geq 8$ ) also did not significantly predict benefit from AAP versus PP [27]. In light of these analyses and considerations, the use of initial diagnostic GS for clinical decision making in mCRPC patients implies relevant limitations and, hence, should not be a key consideration when deciding whether to treat with AAP or not.

The present study is not devoid of limitations, including its post hoc nature. However, it was performed in a prospective randomized clinical trial patient cohort, applying predefined baseline and stratification factors for additional analysis. As we could not confirm significantly different treatment effects based on baseline characteristics, further research is required to establish whether mCRPC patients benefit from early initiation of AAP or other compounds at a disease stage where they are asymptomatic and have low PSA levels, and to determine roles of different systemic treatment options in mCRPC patients.

\section{Conclusions}

This post hoc analysis of the COU-AA-302 study revealed that all patients regardless of baseline characteristics achieved significantly improved outcomes with AAP versus PP. OS, rPFS, and time to chemotherapy and opiate use were longer in asymptomatic mCRPC patients with PSA levels $<80 \mathrm{ng} / \mathrm{ml}$ at the start of treatment and a GS of $<8$ at primary diagnosis compared with patients with advanced PSA or pain levels or a GS of $\geq 8$. Since there was no significantly different treatment effect between both groups, conclusions for clinical decision making cannot be drawn yet. External validation in CRPC patient cohorts is required to establish a potential clinical benefit of early treatment initiation and determine roles of different treatment options in MCRPC patients.

These results have been published in part at the European Urology Association Congress, Munich, Germany, 11-15 March 2016; abstract 775.

Author contributions: Kurt Miller had full access to all the data in the study and takes responsibility for the integrity of the data and the accuracy of the data analysis.

Study concept and design: Diels, Brookman-May.

Acquisition of data: Diels, Brookman-May.

Analysis and interpretation of data: Miller, Carles, Gschwend, Van Poppel, Diels, Brookman-May.

Drafting of the manuscript: Diels, Brookman-May, Miller, Carles, Gschwend, Van Poppel.

Critical revision of the manuscript for important intellectual content: Miller, Carles, Gschwend, Van Poppel, Brookman-May.

Statistical analysis: Diels.

Obtaining funding: None.

Administrative, technical, or material support: None.

Supervision: None.

Other: None.

Financial disclosures: Kurt Miller certifies that all conflicts of interest, including specific financial interests and relationships and affiliations 
relevant to the subject matter or materials discussed in the manuscript (eg, employment/affiliation, grants or funding, consultancies, honoraria, stock ownership or options, expert testimony, royalties, or patents filed, received, or pending), are the following: Kurt Miller has received consultancy fees from Amgen, Astellas, Bayer, Bristol-Myers Squibb, Ferring, Janssen, Medivation, Merck, Merck Sharp \& Dohme, Pfizer, and Roche, and consultancy fees and grants from Novartis. Joan Carles has received consultancy and lecturing fees from Johnson \& Johnson and Bayer, and consultancy fees from Amgen, Astellas, Bristol-Myers Squibb, Pfizer, and Sanofi. Jürgen E. Gschwend has received consulting and lecturing fees from Astellas, Bayer, Janssen, and Roche. Hendrik Van Poppel has no financial disclosures. Joris Diels is an employee of Janssen. Sabine D. Brookman-May is an employee of, and shareholder at, Janssen.

Funding/Support and role of the sponsor: This analysis was funded by Janssen EMEA, which was involved in the design and conduct of the study; collection, management, analysis, and interpretation of the data; and preparation, review, and approval of the manuscript.

Acknowledgments: The authors would like to thank Laurent Antoni (Janssen EMEA) for assistance with reviewing of the manuscript. Writing assistance was provided by Natalie Dennis from PAREXEL, which was funded by Janssen EMEA.

\section{References}

[1] Aziz A, Kempkensteffen C, May M, et al. Prognostic, predictive and potential surrogate markers in castration-resistant prostate cancer. Expert Rev Anticancer Ther 2015;15:649-66.

[2] Yap TA, Zivi A, Omlin A, de Bono JS. The changing therapeutic landscape of castration-resistant prostate cancer. Nat Rev Clin Oncol 2011;8:597-610.

[3] European Medicines Agency. Public statement. Provenge: withdrawal of the marketing authorisation in the European Union. 2015.

[4] Halabi S, Lin CY, Kelly WK, et al. Updated prognostic model for predicting overall survival in first-line chemotherapy for patients with metastatic castration-resistant prostate cancer. J Clin Oncol 2014;32:671-7.

[5] Ryan CJ, Smith MR, de Bono JS, et al. Abiraterone in metastatic prostate cancer without previous chemotherapy. N Engl J Med 2013;368:138-48.

[6] Ryan CJ, Smith MR, Fizazi K, et al. Abiraterone acetate plus prednisone versus placebo plus prednisone in chemotherapy-naive men with metastatic castration-resistant prostate cancer (COU-AA-302): final overall survival analysis of a randomised, double-blind, placebo-controlled phase 3 study. Lancet Oncol 2015;16:152-60.

[7] Scher HI, Halabi S, Tannock I, et al. Design and end points of clinical trials for patients with progressive prostate cancer and castrate levels of testosterone: recommendations of the Prostate Cancer Clinical Trials Working Group. J Clin Oncol 2008;26:1148-59.

[8] Ryan CJ, Kheoh T, Li J, et al. Prognostic index model (PIM) for overall survival (OS) in metastatic castration-resistant prostate cancer ( $\mathrm{mCRPC}$ ) patients (pts) without prior chemotherapy treated with abiraterone acetate (AA) [abstract]. J Clin Oncol 2015;33 (suppl):5047.

[9] Beer TM, Armstrong AJ, Rathkopf DE, et al. Enzalutamide in metastatic prostate cancer before chemotherapy. N Engl J Med 2014;371:424-33.

[10] Armstrong AJ, Garrett-Mayer ES, Yang YC, de Wit R, Tannock IF, Eisenberger M. A contemporary prognostic nomogram for men with hormone-refractory metastatic prostate cancer: a TAX327 study analysis. Clin Cancer Res 2007;13:6396-403.
[11] Halabi S, Vogelzang NJ, Kornblith AB, et al. Pain predicts overall survival in men with metastatic castration-refractory prostate cancer. J Clin Oncol 2008;26:2544-9.

[12] Colloca G, Venturino A, Checcaglini F. Patient-reported outcomes after cytotoxic chemotherapy in metastatic castration-resistant prostate cancer: a systematic review. Cancer Treat Rev 2010;36: 501-6.

[13] Gotay CC, Kawamoto CT, Bottomley A, Efficace F. The prognostic significance of patient-reported outcomes in cancer clinical trials. J Clin Oncol 2008;26:1355-63.

[14] Moreira DM, Howard LE, Sourbeer KN, et al. Predicting time from metastasis to overall survival in castration-resistant prostate cancer: results from SEARCH. Clin Genitourin Cancer 2017;15:60-6.

[15] Schellhammer PF, Chodak G, Whitmore JB, Sims R, Frohlich MW, Kantoff PW. Lower baseline prostate-specific antigen is associated with a greater overall survival benefit from sipuleucel-T in the Immunotherapy for Prostate Adenocarcinoma Treatment (IMPACT) trial. Urology 2013;81:1297-302.

[16] Ryan CJ, Londhe A, Molina A, et al. Relationship of baseline PSA and degree of PSA decline to radiographic progression-free survival (rPFS) in patients with chemotherapy-naive metastatic castration-resistant prostate cancer (mCRPC): results from COU-AA302 [abstract]. J Clin Oncol 2013;31(suppl):5010.

[17] Saad F, de Bono J, Shore N, et al. Efficacy outcomes by baseline prostate-specific antigen quartile in the AFFIRM trial. Eur Urol 2015;67:223-30.

[18] Verzoni E, De Giorgi U, Derosa L, et al. Predictors of long-term response to abiraterone in patients with metastatic castrationresistant prostate cancer: a retrospective cohort study. Oncotarget 2016;7:40085-94.

[19] https://www.g-ba.de/downloads/92-975-218/ 2013-01-14_Modul4A_Abirateronacetat.pdf.

[20] Xu XS, Ryan CJ, Stuyckens K, et al. Correlation between prostatespecific antigen kinetics and overall survival in abiraterone acetatetreated castration-resistant prostate cancer patients. Clin Cancer Res 2015;21:3170-7.

[21] Houede N, Beuzeboc P, Gourgou S, et al. Abiraterone acetate in patients with metastatic castration-resistant prostate cancer: long term outcome of the Temporary Authorization for Use programme in France. BMC Cancer 2015;15:222.

[22] Schlack K, Krabbe LM, Fobker M, Schrader AJ, Semjonow A, Boegemann M. Early prediction of therapy response to abiraterone acetate using PSA subforms in patients with castration resistant prostate cancer. Int J Mol Sci 2016;17:1520.

[23] Nakabayashi M, Hayes J, Taplin ME, et al. Clinical predictors of survival in men with castration-resistant prostate cancer: evidence that Gleason score 6 cancer can evolve to lethal disease. Cancer 2013;119:2990-8.

[24] May M, Brookman-May S, Lebentrau S, et al. Concordance of the Gleason score in prostate multibiopsy and definitive histology. Aktuelle Urol 2010;41:184-92.

[25] Brookman-May S, May M, Wieland WF, et al. Should we abstain from Gleason score 2-4 in the diagnosis of prostate cancer? Results of a German multicentre study. World J Urol 2012;30:97-103.

[26] Melia J, Moseley R, Ball RY, et al. A UK-based investigation of interand intra-observer reproducibility of Gleason grading of prostatic biopsies. Histopathology 2006;48:644-54.

[27] Fizazi K, Flaig TW, Stockle M, et al. Does Gleason score at initial diagnosis predict efficacy of abiraterone acetate therapy in patients with metastatic castration-resistant prostate cancer? An analysis of abiraterone acetate phase III trials. Ann Oncol 2016;27:699-705. 